\title{
Meta
}

Journal des traducteurs

Translators' Journal

\section{Colombie-Britannique : les missionnaires catholiques et les activités langagières (1842-1952)}

\section{Ginette Demers}

Volume 49, numéro 3, septembre 2004

L'histoire de la traduction et la traduction de l'histoire History of Translation and Translation of History

URI : https://id.erudit.org/iderudit/009384ar

DOI : https://doi.org/10.7202/009384ar

Aller au sommaire du numéro

Éditeur(s)

Les Presses de l'Université de Montréal

ISSN

0026-0452 (imprimé)

1492-1421 (numérique)

Découvrir la revue

Citer cet article

Demers, G. (2004). Colombie-Britannique : les missionnaires catholiques et les activités langagières (1842-1952). Meta, 49(3), 656-668.

https://doi.org/10.7202/009384ar
Résumé de l'article

Étant donné la diversité et la difficulté des langues parlées par les Autochtones de la Colombie-Britannique, la plupart des missionnaires catholiques utilisèrent une langue véhiculaire, le Chinook Jargon, pour la prédication et l'enseignement religieux. Cette langue, bien que très répandue, n'était cependant pas comprise par tous les Amérindiens, de sorte qu'ils durent aussi avoir recours à des interprètes. Le présent article fait état des difficultés liées à l'interprétation, particulièrement $\mathrm{au} \mathrm{XIX}^{\mathrm{e}}$ siècle. Les activités de quelques missionnaires illustrent, quant à elles, les réalisations sur le plan langagier : traduction, rédaction, lexicographie, invention d'écritures.
Ce document est protégé par la loi sur le droit d'auteur. L'utilisation des services d’Érudit (y compris la reproduction) est assujettie à sa politique d'utilisation que vous pouvez consulter en ligne.

https://apropos.erudit.org/fr/usagers/politique-dutilisation/ 


\title{
Colombie-Britannique: les missionnaires catholiques et les activités langagières (1842-1952)
}

\author{
GINETTE DEMERS \\ Université Laval, Québec, Canada \\ ngdemers@mediom.qc.ca
}

\begin{abstract}
RÉSUMÉ
Étant donné la diversité et la difficulté des langues parlées par les Autochtones de la Colombie-Britannique, la plupart des missionnaires catholiques utilisèrent une langue véhiculaire, le Chinook Jargon, pour la prédication et l'enseignement religieux. Cette langue, bien que très répandue, n'était cependant pas comprise par tous les Amérindiens, de sorte qu'ils durent aussi avoir recours à des interprètes. Le présent article fait état des difficultés liées à l'interprétation, particulièrement au XIX $x^{e}$ siècle. Les activités de quelques missionnaires illustrent, quant à elles, les réalisations sur le plan langagier: traduction, rédaction, lexicographie, invention d'écritures.
\end{abstract}

\begin{abstract}
Because the different languages spoken by the First Nations of British Columbia were extremely difficult, most Catholic missionaries preached and taught in the Chinook Jargon, a trade language. However, the Jargon was not known to all natives, so they often had to use interpreters as well. This article shows some of the problems the missionaries had with interpreters, especially in the 19th century. Moreover, the linguistic activities of a few missionaries serve to illustrate what was done during the mission era as far as translation, writing, lexicography and invention of alphabets go.
\end{abstract}

\section{MOTS-CLÉS / KEYWORDS}

Colombie-Britannique, missionnaires catholiques, interprétation, Chinook Jargon, invention d'écritures

La première personne qui mit par écrit des mots appartenant à l'une des langues parlées par les Amérindiens de la Colombie-Britannique est le capitaine James Cook. En effet, au cours du voyage de découverte qu'il fit de 1776 à 1779, il passa un mois à Nootka, sur la côte ouest de l'île de Vancouver, et compila un petit vocabulaire anglais-moachat. Entre 1785 et 1800 , bon nombre de marchands qui faisaient la traite des fourrures par mer élaborèrent eux aussi des lexiques bilingues. L'interprétation, quant à elle, joua un rôle non négligeable dès les débuts de la traite et prit une importance considérable pendant la première moitié du xix ${ }^{e}$ siècle dans les comptoirs de la Northwest Company et de la Hudson's Bay Company. Les missionnaires catholiques, arrivés dans la foulée des commerçants de fourrures, se consacreront à l'évangélisation des Amérindiens à partir du début des années 1840. Ils auront à leur tour besoin d'interprètes; certains effectueront un travail de lexicographie beaucoup plus approfondi que ne l'avaient fait les explorateurs et les commerçants de fourrures; ils seront les premiers, enfin, à traduire des textes vers les langues amérindiennes et à donner une écriture aux Autochtones. 


\section{LE CONTEXTE HISTORIQUE}

Il est difficile de savoir quelles étaient les croyances des Amérindiens avant l'arrivée des Européens. En effet, si les explorateurs et les marchands ont parfois décrit leur culture matérielle, ils n'ont pu faire que des suppositions quant à leur religion parce que la connaissance limitée qu'ils avaient de la langue les empêchait de comprendre les idées abstraites. Selon la tradition, ces Autochtones ne faisaient pas de différence fondamentale entre le monde spirituel et le monde matériel, croyaient à l'influence des astres et attribuaient une grande valeur morale au partage (George 1983: 11, 16-18).

En 1789, les Espagnols construisaient à Nootka un petit établissement qu'ils occupèrent jusqu'en 1795. Parmi eux se trouvaient quatre franciscains qui avaient pour mission d'évangéliser les Autochtones, mais d'après le père Patero (cité par Archer 1993: 143), il leur fut impossible de prêcher l'Évangile parce que, le terrain ne se prêtant pas à l'agriculture, ceux-ci ne pouvaient être sédentarisés. Par conséquent, ces religieux eurent peu de contacts avec les Nootkas. Il ne semble pas, non plus, que les pratiques religieuses des Espagnols, dont les Nootkas furent témoins, aient eu une influence sur leur culture spirituelle (Lamirande 1978: 410).

Un peu plus tard, des «prophètes » autochtones, qui avaient manifestement été en contact avec des catholiques, firent leur apparition sur le versant ouest des Rocheuses, prédisant l'arrivée des Blancs et les merveilles que ceux-ci allaient apporter. L'enseignement qu'ils dispensaient d'un village à l'autre consistait en un mélange de croyances amérindiennes et de croyances chrétiennes. Ainsi, ils avaient conservé les danses cérémonielles et d'autres manifestations de la spiritualité amérindienne, mais incitaient également leurs disciples à adorer un Dieu unique, à faire le signe de la croix et à confesser publiquement leurs péchés (Duff 1969: 88-89). Les prophètes ouvrirent la voie aux missionnaires, de sorte que l'enseignement de ces derniers fut généralement bien accueilli.

Au début des années 1830, d’anciens employés de la Hudson's Bay Company pour la plupart des voyageurs ou des interprètes francophones et catholiques - avaient commencé à s'établir dans la vallée de la Willamette, en Oregon ${ }^{1}$, et ils réclamaient des prêtres. Toutefois, c'est seulement en 1838 qu'arrivèrent deux missionnaires, FrançoisNorbert Blanchet et Modeste Demers, envoyés par le diocèse de Québec. Ceux-ci étaient chargés d'une double mission : exercer leur ministère auprès des familles catholiques et évangéliser les Autochtones. L'apostolat de l'abbé Blanchet, qui allait plus tard devenir évêque d'Oregon, s'effectua au sud du $49^{\mathrm{e}}$ parallèle. L'abbé Demers, quant à lui, étendit rapidement ses activités vers le nord, parcourant dès 1842 une partie de la région qui constitue aujourd'hui la Colombie-Britannique. Vers le milieu des années 1850, alors qu'il était évêque de l'île de Vancouver et qu'il avait désespérément besoin de missionnaires, il s'adressa aux Oblats de Marie-Immaculée. Ceux-ci, en effet, se trouvaient en Oregon depuis 1847, mais envisageaient de quitter ce territoire en raison du conflit qui opposait les Autochtones et les pionniers américains. Les oblats établirent une mission à Esquimalt en 1858. D'autres suivirent, et ces religieux occupèrent une place prépondérante dans les missions catholiques pendant une centaine d'années.

Parmi les protestants, ce sont surtout les anglicans et les méthodistes qui s'intéressèrent aux Autochtones de la Colombie-Britannique. Le premier missionnaire anglican, un Britannique, était arrivé à Victoria en 1857. Cinq ans plus tard, il fondait un 
village modèle près de Port Simpson. À la même époque, des ministres méthodistes venus de l'Est du Canada étaient à l'œuvre à Victoria et à Nanaimo².

\section{LES MISSIONNAIRES CATHOLIQUES}

Les activités de quelques missionnaires, prêtres séculiers ou oblats, serviront à illustrer ce qui s'est fait sur le plan langagier au cours de la période étudiée. Toutefois, il convient d'abord de parler d'une langue véhiculaire qui joua un rôle considérable à l'époque des missions.

\section{Le Chinook Jargon}

Comme les langues parlées à l'ouest des Rocheuses étaient extrêmement difficiles, à peu près tous les missionnaires ont d'abord appris le Chinook Jargon, qui était couramment utilisé sur la côte ouest pendant la première moitié du XIX ${ }^{\mathrm{e}}$ siècle et qui s'est ensuite répandu progressivement à l'intérieur des terres. C'est même la seule langue que beaucoup allaient employer dans leurs rapports avec les Amérindiens.

Les chercheurs ne s'entendent pas sur la date d'apparition de ce sabir. Certains estiment que les Autochtones y avaient recours avant l'arrivée des Européens et qu'il était alors composé de mots appartenant à différentes langues amérindiennes. Ces auteurs font valoir le fait que des mots faisant partie du jargon figurent dans un ou deux lexiques anglais-nootka élaborés à la fin du XVIII ${ }^{\mathrm{e}}$ siècle ou au début du XIX ${ }^{\mathrm{e}}$ (Thomas 1970: ix). Cependant, aucun explorateur, aucun commerçant de fourrures n'a soupçonné l'existence de cette langue avant les années 1810. Bien des linguistes croient, d'ailleurs, qu'elle est apparue pendant cette décennie, à la suite des échanges commerciaux entre les Autochtones et les employés de la Northwest Company, laquelle possédait un comptoir à l'embouchure du Columbia. À cette époque, le sabir comprenait des mots amérindiens (chinooks et nootkas, surtout) et des onomatopées de même que des mots anglais et français, souvent déformés (le mot mère, par exemple, y était devenu lamai). Comme les premiers missionnaires étaient francophones, tout le vocabulaire religieux qu'ils y ajoutèrent, à l'exception du mot Dieu (Sahaletaye), fut emprunté au français (Jesu-kri, laconfirmatio, lebatem, legliz, lemaliaj, etc.). La grammaire, quant à elle, était extrêmement simple.

Le grand avantage que présentait cette langue, c'est qu'elle pouvait être apprise en quelques mois. En outre, lorsque les missionnaires devaient travailler auprès de groupes amérindiens qui ne comprenaient pas le chinook, il leur était beaucoup plus facile de trouver une personne qui parlait à la fois chinook et le dialecte de ces Autochtones qu'une personne qui pouvait traduire à partir du français ou de l'anglais ${ }^{3}$.

On peut toutefois se demander s'il s'agissait d'un véhicule adéquat pour transmettre les mystères de la foi. En effet, Modeste Demers déplore le fait que le sabir ne possède pas de participes et qu'un grand nombre de mots aient plusieurs sens. "Il s'ensuit, dit-il, qu'il n'est pas facile de traduire des expressions françaises dans cette langue; il [...] faut utiliser des paraphrases» (cité par Blanchet 1996: 58). AdrienGabriel Morice, un oblat qui œuvra en Colombie-Britannique de 1880 à 1903, estime pour sa part que le chinook fit plus de mal que de bien parce que trop de missionnaires s'en contentèrent plutôt que d'apprendre les langues amérindiennes qui, selon lui, pouvaient rendre de façon beaucoup plus appropriée les enseignements de l'Église 
(1923 : 256). Quoi qu'il en soit, la rareté des commentaires négatifs et la longue utilisation du sabir autorise à penser que, dans l'ensemble, les missionnaires le considéraient comme acceptable.

\section{Le pionnier: Modeste Demers}

Dans ses instructions aux abbés Blanchet et Demers, Mgr Signay, évêque de Québec, leur demandait non seulement de s'appliquer, dès leur arrivée en Oregon, à l'apprentissage des langues amérindiennes, mais aussi d'essayer d'en dégager des principes généraux de façon à pouvoir publier une grammaire au bout de quelques années (cité par Blanchet 1996: 31). Cependant, les missionnaires se rendirent vite compte qu'ils n'arriveraient peut-être jamais à parler la langue des Chinooks (le chinook proprement dit), auprès de qui ils devaient travailler à leur arrivée à l'ouest des Rocheuses. C'est pourquoi ils apprirent plutôt le jargon qui, comme nous l'avons vu, permettait d'instruire un grand nombre d'Autochtones, soit directement, soit par l'intermédiaire d'interprètes 4 .

Dès l'hiver 1838-1839, l'abbé Demers élaborait un lexique chinook-anglais dans le but d'aider ceux qui viendraient après lui. Par la suite, il traduisit en chinook des cantiques, de nombreuses prières et le petit catéchisme. Après son décès, survenu en 1871, son compagnon des premières heures, François-Norbert Blanchet, publia dans un même volume les traductions et le lexique, revu et augmenté.

Le missionnaire a employé dix-huit des lettres de notre alphabet pour la transcription du vocabulaire chinook. Il explique que toutes les lettres d'un mot sont prononcées et que les mots empruntés au français ou à l'anglais sont orthographiés de la façon dont les Autochtones les prononcent (ainsi, médecine y est écrit metsin).

Il n'a donc pas eu recours à un système d'écriture différent du nôtre, même s'il estimait que la prononciation de certains mots - Sahaletaye (Dieu) et hihkt (unique), par exemple - était tellement difficile qu'il avait du mal à trouver des lettres pour la représenter (Blanchet 1996: 58). Cela limitait sûrement l'utilité du lexique. En effet, dans un journal qui prend la forme d'une longue lettre à un ami, l'abbé Jean-Baptiste Bolduc fait figurer une traduction du Pater, en précisant qu'il a souligné les mots dont il est impossible de savoir la prononciation à moins de les avoir entendus (1844: 93). Le tiers des mots sont soulignés.

D’après De Roo (cité par Hanley 1965: 30), Modeste Demers avait fini par apprendre une quinzaine de dialectes amérindiens malgré leur difficulté, et on lui attribue une petite publication de quinze pages intitulée Dictionary of Indian Tongues [...] qui contient des mots tsimshians, haidas et chinooks avec leurs équivalents anglais (Thomas 1970: 168).

\section{Le journal d'Augustin-Joseph Brabant}

Augustin-Joseph Brabant fait partie du petit nombre de prêtres séculiers qui travaillèrent auprès des Autochtones de la Colombie-Britannique au XIX $\mathrm{x}^{\mathrm{e}}$ siècle. Né à Rolleghem en 1845, il fit ses études de théologie au Collège américain de Louvain et fut ordonné prêtre en 1868. Arrivé à Victoria dès 1869, il passa le reste de sa vie sur l'île de Vancouver. C'est lui qui fonda, à Hesquiat, la première mission sur la côte ouest de l'île. Il mourut en 1912. 
L'abbé Brabant ne se distingue par aucune activité langagière remarquable, mais son journal, qui couvre les années 1874 à 1900, est une des rares sources qui fassent mention des problèmes auxquels se heurtaient souvent les missionnaires quand ils devaient recourir à un interprète. Par exemple, il note en 1874, année où il visite divers villages amérindiens en compagnie de Mgr Seghers, qui avait succédé à Modeste Demers :

[18 avril] Our arrival caused a great deal of excitement. Our interpreter had a thundering voice, but we were told he did not translate His Lordship's words with much correctness. Perhaps he thought that shouting would have the necessary effect (1977: 15-16).

[29 avril] Early in the morning we assembled the Indians and began anew to instruct them. When this was done, our Kyuquot interpreter refused to interpret, and gave for his reason that the Checklesets were mocking and insulting him (1977: 19).

[3 mai] The Ehattesaht Indians had come across and joined the Nuchatlahts. [...] Our interpreter was of little account, and our success was not in keeping with the great preparations they had made to receive us (1977:21)

L'année suivante, quand le missionnaire arrive à Hesquiat pour s'y installer, il déplore le fait que personne ne puisse lui servir d'interprète, mais ce n'est peut-être pas mauvais puisque cela le force à apprendre la langue de ses ouailles !

Bien que l'abbé Brabant mentionne parfois sans commentaire la présence d'un interprète et qu'à une occasion il note que son interprète est excellent, les remarques négatives dominent. De façon générale, d'ailleurs, on considère l'interprétation comme un pis-aller qui n'est pas exempt de dangers. Chez les oblats, par exemple, les supérieurs ne cessent d'exhorter les missionnaires à apprendre les langues amérindiennes. «Il n'y a pas le moindre doute que ce qu'on dira soi-même, avec difficulté et d'une manière peu correcte, mais intelligible, sera toujours préféré et préférable à la parole d'un interprète», écrit le père Bermond dès 1858 (cité par Dionne, 1947, p. 21). Il ajoute que "ces sortes de gens», n'ayant souvent qu'une connaissance superficielle de la religion, ne traduisent pas de façon fidèle les paroles des missionnaires et qu'étant malgré tout portés à faire «les petits docteurs et les grands maîtres », ils ont une influence nuisible (ibid.). Une quinzaine d'années plus tard, le père McGuckin affirmera qu'il est impossible de bien instruire les Autochtones par l'intermédiaire d'interprètes, d'autant plus qu'à son avis, un bon interprète est « chose bien rare» (cité par Oblats de Marie-Immaculée 1874: 331).

Tous les missionnaires durent acquérir une certaine connaissance des langues autochtones (autrement, ils n'auraient pas pu entendre les confessions), mais la plupart ne les maîtrisèrent jamais suffisamment pour les utiliser du haut de la chaire: quelle qu'ait été leur opinion des interprètes, ils eurent donc longtemps recours à leurs services.

\section{Adrien-Gabriel Morice: un homme aux multiples talents}

Adrien-Gabriel Morice, né en 1859 à Saint-Marc-sur-Colmont, en France, arrive à New Westminster (Vancouver) en 1880. Envoyé à Williams Lake en 1881, il est d'abord responsable de l'école, puis de 1883 à 1885, sa tâche est d'évangéliser les Chilcotins, tout en continuant d'habiter à la mission. En 1885, on lui confie la mission du lac Stuart, dont il aura la responsabilité jusqu'en 1903. Il quitte peu après la Colombie- 
Britannique pour le Manitoba, où il se consacre surtout à des recherches historiques et lexicographiques, et décède à Winnipeg en 1938.

Le père Morice fut un chercheur passionné et un auteur prolifique. En effet, au cours des années qu'il passa en Colombie-Britannique, il effectua de nombreuses études ethnographiques et anthropologiques, dressa des cartes géographiques, inventa une écriture syllabique, publia un périodique et des livres de lecture en carrier, et traduisit également des textes religieux dans cette langue. Par la suite, il rédigea, entre autres, une histoire de l'Église dans l'Ouest canadien en quatre volumes ainsi qu'un ouvrage de 1500 pages intitulé The Carrier Language ${ }^{5}$.

Ce religieux avait manifestement beaucoup de facilité pour les langues : il apprit rapidement non seulement l'anglais et le chinook, mais aussi le chilcotin et le carrier. À la fin de la première année de son apostolat auprès des Chilcotins, par exemple, il prêchait dans leur langue, avait traduit le catéchisme et des prières, et compilé un petit vocabulaire. L'année suivante, il avait terminé un lexique de 170 pages (Carrière 1972: 340-341).

Avant même de partir pour le lac Stuart, il se lançait dans l'étude du carrier (langue de la plupart des Autochtones qui habitaient son futur territoire), dont il admira vite la richesse et la complexité. Ainsi, il est visiblement émerveillé quand il découvre qu'on n'y trouve pas de générique correspondant au mot casser, mais qu'il existe par contre au moins 110 spécifiques, dont aucun ne peut être substitué à un autre (Mulhall 1986: 43).

Bien que son amour des langues soit évident, une mauvaise expérience explique peut-être aussi en partie le fait que ce missionnaire ait toujours tenu à communiquer directement avec les Amérindiens dont il avait la charge. En effet, il ne pouvait tout apprendre en même temps, et, en 1886, le dialecte des Babines, bien différent de celui qui était parlé dans la région du lac Stuart, ne lui était pas familier. Sa connaissance du chilcotin, par contre, dialecte de la même famille linguistique que celui des Babines, lui permit de se rendre compte que son interprète, qui était censé comprendre le français, était «à peu près nul». Il essaya alors l'anglais, puis le chinook, mais l'interprète ne connaissait pas bien ces langues non plus. Finalement, il se rabattit sur «le français du pays $^{6}{ }$ et réussit à se faire comprendre. Mais il n'arrivait pas à s'habituer à ce jargon et, dès qu'il put balbutier le dialecte des Babines, il s'en servit (Morice 1933: 164-165).

Comme les Carriers étaient disséminés sur un territoire extrêmement vaste, le prêtre estimait que son ministère serait grandement facilité s'ils pouvaient lire leur langue. Selon lui, l'alphabet français ne convenait pas parce qu'il aurait fallu y ajouter 44 lettres pour transcrire tous les sons, ce qui aurait rendu l'apprentissage très fastidieux. Quelques mois après son arrivée au lac Stuart, il tenta donc de trouver un moyen plus simple. L'écriture syllabique que James Evans avait inventée pour les Cris ne comportait pas, non plus, suffisamment de signes pour rendre les sons des dialectes dénés, mais le religieux emprunta à ce missionnaire méthodiste «both the idea of signs representing syllables and the pointing of the signs in different directions to represent sound changes» (Mulhall 1986: 45).

L'écriture que le père Morice élabora, son «syllabaire méthodique» comme il l'appelait, était plus complexe que celle d'Evans, mais il considérait qu'elle était plus facile à apprendre parce que ses éléments, regroupés de façon plus rationnelle, découlaient en quelque sorte les uns des autres et qu'aucun signe ne portait à confusion. Les Carriers adoptèrent très vite le syllabaire: le missionnaire passa une semaine dans 
chacun des quatre villages les plus importants et l'enseigna à des jeunes gens. Ceuxci l'enseignèrent à leur tour à d'autres. Les Autochtones se mirent bientôt à communiquer les uns avec les autres en traçant les signes dans le sable ou en écrivant avec du charbon sur des arbres dont ils avaient enlevé l'écorce (ibid.).

Après avoir acheté une presse, le père Morice fit fondre des caractères avec lesquels il imprima des livres de lecture de même que ses traductions de textes liturgiques et pastoraux. En outre, à partir de 1891, il publia un périodique mensuel intitulé Test' les nahivelnek (Le papier qui raconte), rédigé lui aussi en écriture syllabique. On trouvait dans chaque numéro des nouvelles locales, régionales et même internationales, un extrait de l'Évangile, la vie d'un saint, un article portant sur l'histoire naturelle ou la géographie, etc. Selon Carrière (1972: 327), le journal parut d'octobre 1891 à juin 1894, mais Mulhall (1986: 145) laisse entendre qu'il existait toujours en $1899^{7}$.

Le missionnaire s'intéressait également à la culture des Amérindiens qui peuplaient l'intérieur de la Colombie-Britannique: il fut le premier à consigner par écrit et à traduire des échantillons de leur littérature orale. Ainsi, en 1895, il publiait en anglais trois légendes des Carriers. Dans l'introduction, il explique sa façon de procéder: il demandait d'abord à un Autochtone «fiable» de lui raconter la légende, qu'il tentait ensuite de répéter verbatim, et le conteur le corrigeait s'il se trompait. Puis il écrivait le texte en carrier et le lisait à haute voix pour s'assurer de nouveau qu'il n'y avait pas d'erreurs. Finalement, il effectuait la traduction. Il reconnaît que cette méthode peut sembler peu rigoureuse, mais souligne que, maîtrisant le carrier aussi bien que sa langue maternelle, il pouvait sans difficulté répéter et transcrire fidèlement ce qu'on lui avait dit. À son avis, cela permettait de mieux rendre le style que si la légende avait été dictée (Morice 1895: 3-4).

Ajoutons que le père Morice traduisit aussi en français certains de ses articles et un de ses ouvrages qui avait d'abord paru en anglais.

Parallèlement à ses activités de rédaction et de traduction, il menait des recherches anthropologiques qui firent l'objet de nombreuses publications. Le mérite de ces travaux est qu'à une époque où la plupart des américanistes amateurs élaboraient des théories fantaisistes pour expliquer l'origine de la culture des Autochtones, le prêtre se fondait sur la langue (Mulhall 1986: 146). Cette approche linguistique lui a d'ailleurs permis d'arriver à des conclusions dont certaines restent valables aujourd'hui.

C'est au cours des années passées au lac Stuart, enfin, que le missionnaire recueillit les renseignements qui allaient servir à l'élaboration de son ouvrage monumental sur le carrier. Il s'agit en partie d'un dictionnaire carrier-anglais, mais on y trouve aussi de multiples observations morphologiques, grammaticales, syntaxiques, phonétiques et ethnographiques, de même que des textes en carrier - quatre légendes et des extraits du catéchisme - accompagnés de leur traduction en anglais. Le verbe y est traité de façon particulièrement détaillée, et les difficultés sont illustrées par de nombreux exemples.

Edward Sapir fit un compte rendu élogieux de cette étude, la qualifiant de «magnificent work» et disant qu'à sa connaissance, «the problem of handling grammar and vocabulary as a formal unit has never been before attacked in so original a manner nor with so sure an instinct» (1935: 500-501).

Bien d'autres travaux d'Adrien-Gabriel Morice connurent un succès durable, mais, à sa grande déception, son écriture syllabique fut rapidement détrônée par la sténographie Duployé, adaptée aux langues autochtones par Jean-Marie Lejeune, un oblat qui se trouvait en Colombie-Britannique à la même époque. 


\section{Jean-Marie Lejeune: traducteur, lexicographe, rédacteur}

Jean-Marie Lejeune, un Breton, naquit en 1855. Ordonné prêtre en 1879, il partit la même année pour la Colombie-Britannique. Après un bref séjour à New Westminster, il travailla auprès des Autochtones de Mission City, de Sechelt, de Stalo et de Yale (1880-1882). Il fut ensuite envoyé à Kamloops où il demeura jusqu'en 1929, s'occupant des Thompsons, des Nicolas et des Shushwaps. Il mourut à New Westminster l'année suivante.

Quand il s'était rendu en Colombie-Britannique, le père Lejeune avait fait le voyage en compagnie de Paul Durieu, futur évêque de New Westminster, qui lui avait enseigné le chinook. Bien qu'il ait appris par la suite plusieurs dialectes amérindiens, il eut toujours un intérêt particulier pour le sabir, "that wonderful language», selon l'expression qui figure dans le titre du petit lexique qu'il publia en 1886.

D’après lui, si une très bonne connaissance du chinook permettait de transmettre efficacement sa pensée, le fait que cette langue n'était pas comprise par tous les Autochtones posait problème parce qu'il fallait alors recourir à des interprètes qui, souvent, préféraient dire quelque chose qui n'avait aucun rapport avec le texte plutôt que d'avouer qu'ils n'avaient pas compris un passage. Il conseillait donc de prendre le temps d'expliquer à l'interprète ce qui allait être dit avant de s'adresser aux Amérindiens. Lui-même, toutefois, s'efforça d'apprendre rapidement leurs langues. Gurney (1948: 63) va jusqu'à dire que son extrême méfiance des interprètes a probablement contribué au fait qu'il soit devenu un virtuose des dialectes autochtones!

À son arrivée à New Westminster en 1879, ce religieux constata que certains des missionnaires qui l'avaient précédé avaient tenté, en vain, d'utiliser des caractères syllabiques pour mettre par écrit certains des dialectes parlés à l'ouest des Rocheuses (Gurney 1948: 108). Il se mit lui aussi à la tâche, essayant d'adapter l'écriture de James Evans au chinook et à quelques dialectes. Selon cette méthode, toutefois, il était nécessaire d'ajouter un signe à la fin des syllabes qui se terminaient par une consonne. Or, les langues parlées dans le sud de la Colombie-Britannique contenaient beaucoup de consonnes. Par conséquent, pour écrire un mot comme mokst, il aurait fallu ajouter trois signes. Dans le cas de $t-k w a-k o u l-t k-s n ' t n$, un mot shuswap, le père Lejeune considéra que cela créait des difficultés insurmontables. Il abandonna à son tour la partie, trouvant plus simple d'employer notre alphabet.

En 1890, après avoir étudié l'écriture du père Morice, le missionnaire constatait qu'elle ne pouvait pas non plus être utilisée, toujours en raison de «la multiplicité des consonnes» (s.d.: 4575). C'est alors qu'il eut l'idée de la sténographie inventée par l'abbé Émile Duployé, qu'il avait apprise à l'âge de seize ans. Après y avoir apporté quelques modifications, il se rendit compte qu'elle pouvait tout à fait convenir à la transcription du chinook et des dialectes qu'il connaissait. La sténographie présentait plusieurs avantages: elle s'apprenait rapidement, elle était universelle et elle pouvait faciliter l'apprentissage de l'anglais chez les Amérindiens. Elle eut un immense succès tant auprès des missionnaires que des Autochtones: utilisée d'abord dans la région de Kamloops, elle se répandit vite jusqu'à l'embouchure du Fraser, puis le long du littoral et finalement sur l'île de Vancouver (Cronin 1960: 171; Nicolaye à Lejeune, 28 août 1899).

En 1891, le père Lejeune imprimait et diffusait un document de 32 pages intitulé Elements of Shorthand. Part I. L'année suivante, un livre de lecture en chinook était 
tiré à 1300 exemplaires. En 1894, 2000 Amérindiens pouvaient lire et écrire la sténographie. Ils se mirent à correspondre non seulement les uns avec les autres, mais aussi, dans certains cas, avec des personnes de l'Est du Canada, des États-Unis et même d'Europe ${ }^{8}$ qui connaissaient à la fois le chinook et la sténographie.

La plupart des publications de Jean-Marie Lejeune sont écrites en sténo. Il s'agit surtout de prières, de cantiques et du catéchisme traduits en divers dialectes. Un ouvrage digne de mention est son Poliglott [sic] Manual, publié par sections de 1896 à 1899. Cet ouvrage multilingue de 550 pages $^{9}$ rédigé presque entièrement en écriture sténographique (la section anglaise fait exception) comprend des prières et le catéchisme en anglais, les prières de la messe en latin ainsi que des prières, des cantiques et le catéchisme en chinook et dans les dialectes suivants: stalo, thompson, lillooet, okanagan, shuswap, squamish, sechelt et slayamen.

Le Kamloops Wawa, un périodique qu'il fonda en 1891, suscita un intérêt considérable. Il fut en effet recherché par des organismes prestigieux tels que le Smithsonian Institute et le British Museum; en outre, la Provincial Library of British Columbia, la New York State University et l'Université Laval y étaient abonnées (Gurney 1948: 139).

Le Wawa parut chaque semaine jusqu'en 1893, une fois par mois de 1894 à 1900 et quatre fois par année de 1901 à 1904. (Les souvenirs du père Lejeune figurent dans des numéros spéciaux, rédigés en français et tirés à un petit nombre d'exemplaires, qui furent publiés mensuellement en 1915, 1916 et 1917.) Au début, il était écrit uniquement en chinook, mais une section anglaise fut ajoutée par la suite. On y trouvait généralement une leçon de sténographie, des extraits de textes religieux ou pieux, des avis de naissance, de mariage et de décès de même que des nouvelles.

Pendant les premières décennies du $\mathrm{xx}^{\mathrm{e}}$ siècle, un nombre croissant d'Amérindiens apprenaient à lire et à écrire l'anglais : l'utilité de la sténographie diminuait, et le père Lejeune n'eut plus que rarement recours à cette forme d'écriture.

\section{François-Marie Thomas et l'interprétation}

Cet oblat, né en Bretagne en 1868, fut envoyé en Colombie-Britannique en 1894. Il passa trois ans à New Westminster où Paul Durieu le forma, puis 55 ans à la mission Saint-Joseph, à Williams Lake, où il était responsable des Shuswaps, des Carriers et des Chilcotins. Il mourut en 1957.

Le père Thomas avait une grande admiration pour les méthodes d'évangélisation de Mgr Durieu ${ }^{10}$ et il s'y accrocha longtemps après qu'elles eurent été abandonnées par la plupart des missionnaires, qui les considéraient comme désuètes. Il permet ainsi d'illustrer un phénomène inhabituel: le fait que l'interprétation peut empêcher la communication.

Même s'il parlait shuswap et chilcotin, ce religieux préférait employer le chinook et communiquer avec les Autochtones par l'intermédiaire d'un interprète, façon de faire préconisée par Mgr Durieu. Il tenait également à ce que les jeunes prêtres qui le secondaient apprennent cette langue et aient, comme lui, recours à l'interprétation. L'un d'entre eux, John Hennessy (cité par Whitehead 1981:34), explique qu'il aurait aimé prêcher en anglais, ainsi que les Amérindiens le souhaitaient, mais que le père Thomas ne le permettait pas:

He insisted I preached in Chinook and the reason he gave was that amongst the Indians, if you were a man in authority, you always talked through an interpreter. He said 
that this was the way of the great chiefs. That even if they spoke their own language, they would? speaking to the people? speak through an interpreter. So I learnt Chinook $[\ldots]$.

En 1934, année où le père Hennessy était arrivé à Williams Lake, seuls les gens âgés comprenaient encore le chinook. C'était donc surtout les vieux chefs qui servaient d'interprètes. Le missionnaire émet des réserves tant sur le chinook que sur l'interprétation. Il dit en effet s'être vite rendu compte que le sabir permettait difficilement de faire comprendre adéquatement les enseignements de l'Église, en partie parce que le vocabulaire religieux était composé de mots français qui n'avaient pas d'équivalents dans les langues autochtones. Selon lui, les Amérindiens, y compris l'interprète, ne savaient pas ce que ces mots voulaient dire. Pour illustrer son propos, il raconte l'anecdote suivante:

I remember on one occasion the Chief, old Charlie Boy at Redstone, was interpreting [...] and he stopped and he said to me, "What the hell does that word 'la grâce' mean?" This is in church, before everybody. And he had been interpreting for Father Thomas since 1897 probably, at least he'd been hearing it. And this was 1935 ! Almost 40 years. I often wonder just how much we got across to those people you know, in regard to the knowledge of the Faith (ibid.: 34-35).

Une dizaine d'années plus tard, Alex Morris, qui assistait le père Thomas dans son travail auprès des Shushwaps et des Carriers, constatait que le vieil oblat n'avait pas changé: le recours au chinook et à l'interprétation était toujours de rigueur. D'après lui, cette façon de faire était dépassée parce que, dans les missions, l'anglais était la langue parlée par la plupart des Autochtones. Ainsi, il dit qu'un dimanche, alors qu'il remplaçait le père Hennessy à la mission d'Alexandria, il décida de faire son sermon en anglais et que cette initiative fut très appréciée, particulièrement par les jeunes, qui ne comprenaient ni le chinook ni le carrier! Il ajoute qu'à son avis, le manque de communication était depuis longtemps un problème et que cela avait beaucoup nui aux relations entre les missionnaires et les Amérindiens (ibid.: 75).

Après le décès du père Thomas (et bien avant, quand ils pouvaient le faire impunément!), les jeunes missionnaires allaient se rapprocher des Amérindiens, conversant et discutant avec eux de façon à s'assurer qu'ils comprenaient ce qui leur était enseigné. Avec la généralisation de l'anglais et la disparition du régime Durieu, l'importance de l'interprétation et de la traduction religieuse vers les langues amérindiennes décrut rapidement.

\section{CONCLUSION}

L'aspect le plus original des activités langagières à l'époque des missions catholiques en Colombie-Britannique est sans doute la place considérable qu'occupa une langue véhiculaire. En effet, le chinook fit l'objet de plusieurs lexiques, constitua la langue d'arrivée de multiples traductions et fut pendant de nombreuses années la langue de l'instruction religieuse et de la prédication. De façon très générale, c'est également à partir de cette langue, plutôt qu'à partir de l'anglais ou du français, que se faisait l'interprétation vers les langues autochtones.

Bien que l'Ancien et le Nouveau Testament, l'histoire de l'Église catholique et certains exercices de piété aient été traduits en chinook, les textes traduits dans le 
plus grand nombre de dialectes sont le catéchisme, les prières « usuelles » et des cantiques. Il semble donc que seules les connaissances élémentaires aient été largement diffusées.

Pour évaluer la qualité des traductions, il faudrait connaître le chinook et les langues amérindiennes parlées à l'ouest des Rocheuses. Remarquons simplement que, chez les oblats, on insistait sur l'uniformité d'un traducteur à l'autre et d'un dialecte à l'autre. Ainsi, en 1862, des missionnaires traduisent le catéchisme dans cinq des dialectes parlés dans la région de New Westminster, et le père d'Herbomez, alors supérieur, demande au père Fouquet de s'entendre au moins avec le père Grandidier pour qu'il y ait « uniformité dans l'enseignement même de la lettre, comme dans la doctrine» (D'Herbomez à Fouquet, 9 octobre 1862). Une lettre de Paul Durieu montre également que, dans les cas où plus d'un missionnaire parlait la langue d'arrivée, les traductions étaient révisées. L'évêque y mentionne le fait que le père Lejeune a corrigé et annoté une traduction du père Lejacq. En outre, il a lui-même revu une traduction du père Lejeune et estime qu'il y a «quelques corrections à faire » (Durieu à Lejeune, 28 octobre 1894).

Les réalisations d'Adrien-Gabriel Morice et de Jean-Marie Lejeune sont évidemment exceptionnelles. Toutefois, l'invention de deux écritures et la publication de deux journaux ont permis à certains des Autochtones non seulement d'approfondir leur connaissance de la religion, mais aussi d'avoir une ouverture sur le reste du pays et même sur le monde.

Entre 1842, année où Modeste Demers effectuait un voyage de neuf mois dans la partie continentale de la Colombie-Britannique, et 1952, année où François-Marie Thomas prenait sa retraite, l'interprétation joua un rôle très important, soit parce que les missionnaires ne maîtrisaient pas les langues parlées dans le territoire qu'ils desservaient, soit pour des raisons de prestige, la mentalité du XIX ${ }^{\mathrm{e}}$ siècle - selon laquelle le missionnaire devait être considéré comme un grand chef - ayant dans certains cas tardé à disparaître. Il faut reconnaître que les missionnaires n'avaient pas une très haute opinion des interprètes. Avant 1900, en particulier, il semble qu'il s'agissait souvent d'Amérindiens convertis depuis peu : il n'est donc pas étonnant qu'ils n'aient pas toujours compris ce qu'il leur fallait traduire. Néanmoins, quelle qu'ait été la qualité de l'interprétation, l'instruction religieuse dispensée par l'intermédiaire des interprètes ne paraît pas avoir donné de trop mauvais résultats: le recensement de 1939 montre que près de $100 \%$ des Autochtones de la Colombie-Britannique avaient embrassé le christianisme et que la proportion de catholiques atteignait $57 \%{ }^{11}$ (Duff 1969: 87).

\section{NOTES}

1. Avant 1846, année où fut déterminée la frontière actuelle entre la Colombie-Britannique et les ÉtatsUnis, les activités de la compagnie à l'ouest des Rocheuses s'étendaient jusqu'à la Californie.

2. La bibliographie compilée par Lowther (1968) indique que les activités langagières des missionnaires protestants sont comparables dans l'ensemble à celles des missionnaires catholiques. La seule différence notable, c'est que l'ouvrage le plus traduit semble être la Bible, ce qui n'est pas le cas chez les catholiques.

3. En 1875, par exemple, le chinook était compris par 100000 personnes, des Autochtones pour la plupart (Thomas 1970: ix).

4. Dans les débuts, il arrivait souvent qu'une triple ou même une quadruple interprétation soit nécessaire (Association de la propagation de la foi $1841: 72$ ). 
5. Une bibliographie des écrits du père Morice, tant publiés qu’inédits, figure dans Carrière (1972: 325-341).

6. Il s'agit de la langue parlée à cette époque par les Métis qui étaient au service de la Hudson's Bay Company. Le vocabulaire était français, mais la syntaxe était celle des langues autochtones (Mulhall 1986: 53).

7. En effet, cet auteur mentionne qu'en 1898-1899, le missionnaire était très occupé, entre autres, par la rédaction du périodique.

8. Les Européens étaient surtout des novices oblats.

9. Gurney (1948: 64) estime que le nombre de pages s'élèverait à 2200 si notre alphabet avait été utilisé.

10. Paul Durieu prônait l'établissement de villages modèles, qui étaient largement administrés par les Autochtones, mais dans lesquels le prêtre était l'autorité suprême. D'après Whitehead (1981: 29), bien que certains missionnaires aient tenté d'implanter le « régime Durieu » avant l'arrivée du père Thomas, c'est surtout lui qui s'employa à le faire accepter et respecter.

11. À la fin des années 1960, le pourcentage était à peu près le même: 22000 Amérindiens sur 40000 (Duff 1969: 92). La plupart des autres étaient anglicans ou méthodistes.

\section{RÉFÉRENCES}

\section{Sources manuscrites}

\section{ARCHIVES DESCHÂTELETS :}

D’Herbomez à Fouquet (9 octobre 1862) : Collection Oregon et Colombie-Britannique, Dossier D’Herbomez, HPK 5282.H53L34.

Durieu à Lejeune (28 octobre 1894): Collection Oregon et Colombie-Britannique, Dossier Durieu, HPK 5242.D96L104.

Lejeune, J.-M. (s.d.) : «Comment la sténographie a été introduite chez les Sauvages», Fonds St. Peter's, Dossier Lejeune, P 4573-4587.

Nicolaye à Lejeune (28 août 1899) : Fonds St. Peter's, P 4885.

\section{Sources imprimées et sources secondaires}

Archer, C. E. (1993) : «Seduction before Sovereignty: Spanish Efforts to Manipulate the Natives in Their Claims to the Northwest Coast», From Maps to Metaphors. The Pacific World of George Vancouver, Robin Fisher et Hugh Jonston (dir.), Vancouver, UBC Press, p. 127-159.

Association de la Propagation de la Foi (1841): Rapport sur les missions du diocèse de Québec, n³, Québec, Fréchette.

Blanchet, F.-N. (1996): "Scènes de l'histoire de l'Église catholique en Orégon», Les débuts de l'Église catholique en Orégon, Rimouski, Association des familles Blanchet, p. 11-145.

Bolduc, J.-B. Z. (1844) : Mission de la Colombie. Lettre et journal de Mr. J.-B. Z. Bolduc, missionnaire de la Colombie, Québec, Imprimerie J.-B. Fréchette.

Boucher, M. (1983): "The Roman Catholic Church in British Columbia», Circle of Voices. A History of the religious Communities of British Columbia, Charles P. Anderson, Tirthankar Bose et Joseph I. Richardson (dir.), Lantzville, B. C., Oolichan Books, pp. 167-194.

Brabant, A.-J. (1977) : Mission to Nootka. 1874-1900. Reminiscences of the West Coast of Vancouver Island, édité par Charles Lillard, Sidney, British Columbia, Gray's Publishing Ltd.

CAmpbell, L. (1997): American Indian Languages. The Historical Linguistics of Native America, New York-Oxford, Oxford University Press.

CArrière, G. (1972) : «Adrien-Gabriel Morice, o.m.i. (1859-1938). Essai de bibliographie», Revue de l'Université d'Ottawa, vol. 42, p. 325-341.

Cronin, K. (1960): Cross in the Wilderness, Vancouver, Mitchell Press.

Demers, M. (1862): Dictionary of Indian Tongues. Containing Most of the Words and Terms Used in the Tsimpsean, Hydah \& Chinook, with Their Meaning or Equivalent in the English Language, Victoria, Hibben \& Carswell.

Demers, M. (1871): Chinook Dictionary, Catechism, Prayers and Hymns composed in 1838 \& 1839 by Modeste Demers: Revised, Corrected and Completed, in 1867 by F. N. Blanchet; with Modifications and Additions by L. N. St. Onge, Montréal, s. é. 
Dionne, G. (1947): Histoire des méthodes missionnaires utilisées par les Oblats de Marie-Immaculée dans l'évangélisation des Indiens du Versant Pacifique au XIX' siècle, thèse de maitrise, Université d'Ottawa.

Duff, W. (1969): The Indian History of British Columbia, Victoria, Royal British Columbia Museum.

Fowler, R. (1992): «The Contribution of Adrien-Gabriel Morice to Linguistic Ethnology: A Contextual Evaluation», Western Oblate Studies 2, Raymond Huel (dir.), p. 39-50.

George, G. (1983): "Native Indian Spirituality», Circle of Voices. A History of the Religious Communities of British Columbia, Charles P. Anderson, Tirthankar Bose et Joseph I. Richardson (dir.), Lantzville, B.C., Oolichan Books.

Gurney, W. H. (1948): The Work of Reverend Father J. M. R. Le Jeune, O. M. I., thèse de maîtrise, University of British Columbia.

Hanley, P. (1965): The Catholic Ladder and Missionary Activity in the Pacific Northwest, thèse de maîtrise, Université d'Ottawa.

Lamirande, É. (1978) : «Traditions orales du XIXe siècle sur la présence de prêtres espagnols en Colombie-Britannique", Revue de l'Université d'Ottawa, vol. 48, p. 393-413.

Lejeune, J.-M. (1886): Practical Chinook Vocabulary, Comprising All \& the Only Usual Words of That Wonderful Language [...], Kamloops, s.é.

Lejeune, J.-M. (1891): Elements of Shorthand. Part I, Kamloops, s.é.

Lejeune, J.-M. (1896-1899) : Polyglott [sic] Manual, Kamloops, s.é.

Lowther, B. J. (1968): A Bibliography of British Columbia: Laying the Foundations 1849-1899, Victoria, University of Victoria.

Morice, A.-G. (1895): «Three Carrier Myths. With Notes and Comments by the Rev. Father Morice, O.M.I. », Transactions of the Canadian Institute, vol. 5, p. 1-36.

Morice, A.-G. (1923): Histoire de l'Église catholique dans l'Ouest canadien. Du lac Supérieur au Pacifique (1659-1915), vol. 4, Saint-Boniface, chez l'auteur - Montréal, Granger Frères.

Morice, A.-G. (1932): The Carrier Language, 2 vol., Mödling Bei Wien, Österreich, Verlag Der Internationalen Zeitschrift «Anthropos».

Morice, A.-G. (1933): Souvenirs d'un missionnaire en Colombie-Britannique, Winnipeg, Éditions de la Liberté.

Mulhall, D. (1986): Will to Power. The Missionary Career of Father Morice, Vancouver, University of British Columbia Press.

Oblats De Marie-Immaculée (1874): Missions de la congrégation des Oblats de Marie-Immaculée 47.

SAPIR, E. (1935): «The Carrier Language (Déné Family), a Grammar and Dictionary Combined», American Anthropologist, vol. 37, p. 500-501.

Thomas, E. H. (1970): Chinook. A History and Dictionary of the Northwest Coast Trade Jargon, Portland, Oregon, Binfort \& Mort.

Whitehead, M. (1981): Now You Are My Brother. Missionaries in British Columbia, Victoria, Provincial Archives of British Columbia. 